\title{
Application of Statecharts in Buck-Boost DC-DC Converter Simulation
}

\author{
MARCELO G. CENDOYA, GRACIELA M. TOCCACELI \\ Instituto de Investigaciones en Electrónica, Control y Procesamiento de Señales - LEICI \\ Departamento de Electrotecnia, Facultad de Ingeniería, \\ Universidad Nacional de La Plata. \\ CONICET. \\ C.C. 91, La Plata 1900, Buenos Aires. \\ ARGENTINA
}

\begin{abstract}
A very attractive and relatively simple option to simulate a DC-DC converter is to use a hybrid model. In this case, the need to use very small simulation steps (as those necessary to simulate models carried out at the physical level) is avoided. Furthermore, unlike the averaged state-space models, it has the advantage that the model is valid throughout the entire working range of the converter and for both conduction modes (continuous and discontinuous). By simulating several renewable energy conversion systems incorporating DCDC converters, the authors have discovered the true potential of this modeling method. Despite its inherent advantages, this approach to DC-DC converters simulation is not as widely adopted as it should be. This work tries to encourage the reader of its use in certain typical situations.

In this article the implementation of the hybrid model of the DC-DC Buck-Boost converter, using Statechart techniques, is performed. This model was written in the Stateflow language, a tool from the MATLAB ${ }^{\circledR} /$ Simulink environment, and allowed the creation of a building block formed by the described converter model with adequate interfaces to the SimPowerSystem and Simulink environments. The block is validated by comparing simulation results, realized under different operating conditions, with calculations done employing well-known and proven formulas. As an example of the use of the presented block, a buck-boost DC-DC converter with voltage and current control loops is simulated, corroborating its correct performance.
\end{abstract}

Key-Words: - Statecharts; Hybrid models; Buck-boost DC-DC converter; Simulation.

Received: January 7, 2021. Revised: June 23, 2021. Accepted: July 12, 2021. Published: July 31, 2021.

\section{Introduction}

DC-DC converters are used in many applications. Two applications that are of great relevance today are renewable energy conversion systems and electric vehicles. During the design stage of a DCDC converter a very powerful tool is computer simulation.

There are different types of models that can be used for the simulation of DC-DC converters. One option is the use of an electrical model in which the electronic devices that implement the converter switches are represented by complex equivalent subcircuits derived from semiconductor physics theory. In other words, they are models made at physical level. Examples of these are the wellknown, PSPICE and PSIM simulation packages [1] [2]. This type of model allows analyzing in detail the commutation process between switches and diodes, to design gate drive circuits, studying effects of the inverse recovery time of diodes, evaluating the performance of snubber circuits, quantifying switching and conduction losses, etc. .

The simulation of these models involves the use of variable-step numerical integration routines to solve the non-linear differential equations of the model. To achieve the convergence of the numerical integration algorithms during the complex commutation process, extremely reduced simulation steps are required, due to the abrupt changes of the electrical variables in a very short time-lapse. This leads to long simulation times due to the necessary computational cost. This fact makes the use of models at physical level of the DC-DC converter not advantageous in some situations. At least these two very common cases can be mentioned:

(i) When the converter being part of a complex system, with subsystems of much slower dynamics than the converter itself (for example, renewable energy conversion systems). In this case, the simulation will require a lot of computation time to observe a significant temporal evolution of the slower subsystem, since the simulation step will be 
imposed by the fast DC-DC converter commutations.

(ii) During the tuning process of the controllers for voltage and/or current regulation in closed loop systems. In this case it is useful to be able to represent the controller by means of a mathematical function and not by means of an equivalent electrical circuit. At this stage of the process, the control engineer tries to find the most suitable control algorithm, since he is in a design stage prior to the practical implementation of the controller.

For the situation (ii), models have been developed based on the technique of averaging the state-space variables [3]. To be useful, these models must be subsequently linearized. Although they are widely used in the industry with good results, they have two disadvantages: (a) the model is valid in a narrow working range and (b) two different models are needed depending on the converter operation mode (continuous or discontinuous), which represents a great inconvenience [4]. Therefore, to check the overall performance of the control system, repeated simulations must be carried out at various operating points, with different values of load and disturbances, changing the model as appropriate to each case, which represents a very annoying drawback.

In the above mentioned situations, (i) and (ii), it is convenient to use models that retain the character of the converter (a variable structure system, not averaged), but do so based on the ideal switch concept with zero commutation times. In this sense, a very attractive alternative for modeling DC-DC converters is to treat it as a Hybrid System [5] [6] [7] [8] [9] [10] [11]. A Hybrid System is one formed by a discrete subsystem managed by events (or reactive subsystem), and a continuous subsystem. Both subsystems communicating with each other through commands and events. This technique allows, by means of a relatively simple model, to simulate converters with a very low computational effort since the integration method used in solving the equations can work with a fixed step and not as small as in the case of the physical modeling of the semiconductor devices. The modeling of the reactive subsystem of the Hybrid System can be implemented using directed graph techniques (Grafcet, Petri nets, etc). One of the most used is Statecharts, a tool in the MATLAB $\AA /$ Simulink environment, which is programmed in the Stateflow language [12]. This allows a modular, hierarchical and structured programming, grouping states into superstates, providing independence between them (parallelism) and allowing the refinement of states.
It allows creating a model as a generalized state machine.

In this article the hybrid model of the buck-boost DC-DC converter is presented, implementing the reactive part with the Statechart tool, using the Stateflow language and the continuous part using library blocks of the MATLAB ${ }^{\circledR} /$ Simulink environment. A building-block was made consisting of the described model of the converter, with an interface to the SimPowerSystem and MATLAB ${ }^{\circledR} /$ Simulink environments. The validity of the proposed block is made by comparing steadystate simulation results of a buck-boost DC-DC converter in open-loop operation and in the two conduction modes (continuous and discontinuous) with manual calculations. Furthermore, an example of the use of the proposed block is presented to simulate a Buck-Boost DC-DC converter with an external output voltage control loop and an internal average input current control loop.

\section{Analysis of the Buck-Boost DC-DC converter}

To obtain the hybrid model of the converter, we must first review its working principle and then define the possible operating states.

\subsection{Review of the converter operation}

The circuit diagram of the Buck-Boost DC-DC converter is shown in figure 1 (a). It is made up of a controlled switch (S), a diode, an inductor and an output capacitor. The converter is powered by an ideal voltage source $\left(\mathrm{V}_{\mathrm{i}}\right)$ and feeds a load represented by its equivalent resistance (R). When the switch is closed (during ton= $\mathrm{ON}$ time) the diode is reversely biased, the inductor stores energy $\left(\mathrm{i}_{\mathrm{L}}>0\right)$ and the capacitor discharges $\left(\mathrm{i}_{\mathrm{C}}<0\right)$ through the load resistance, maintaining and approximately constant output voltage. When the switch is open (during toff $=$ OFF time) the inductor delivers its stored energy recharging the capacitor $\left(\mathrm{i}_{\mathrm{C}}>0\right)$. If the switch is kept open for a long time, the inductance will run out of stored energy the stream will be extinguished $\left(i_{L}=0\right)$, thereupon the diode does not conducts anymore.

\subsection{States of the buck-boost converter}

The discussion carried out previously allows us to establish that, depending on the conduction situation of the switch and the diode, it is possible to define three states in the Buck-Boost DC-DC converter operation: 
- On state: when the switch is On. The equivalent circuit corresponding to this state is shown in figure 1 (b).

- Offc state: the switch is Off and there is energy stored in the inductor $\left(\mathrm{i}_{\mathrm{L}}>0\right)$, which causes the diode to be forward biased. The equivalent circuit corresponding to this state is shown in figure 1 (c).

- Offnc state: the switch is open and the inductance does not have any stored energy $\left(i_{L}=0\right)$, so the diode is not conducting. The equivalent circuit corresponding to this state is shown in figure 1 (d).

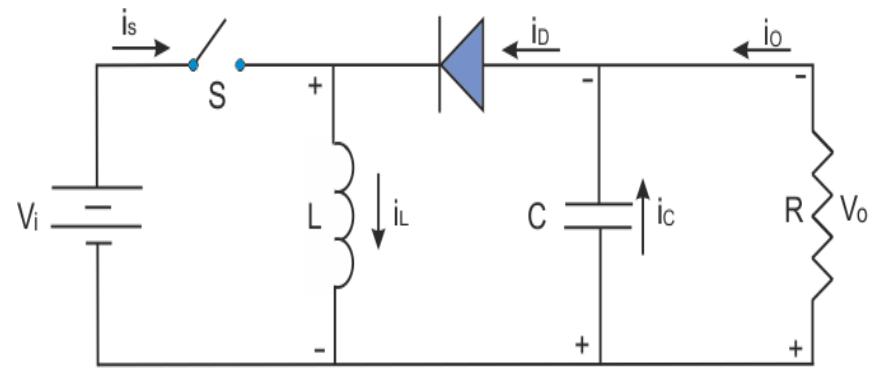

(a)

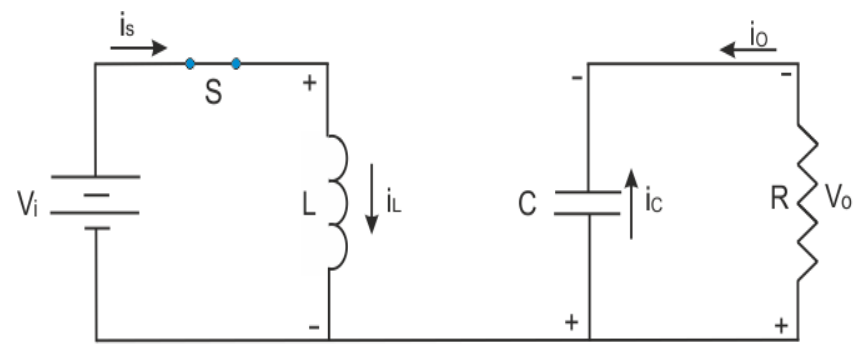

(b)

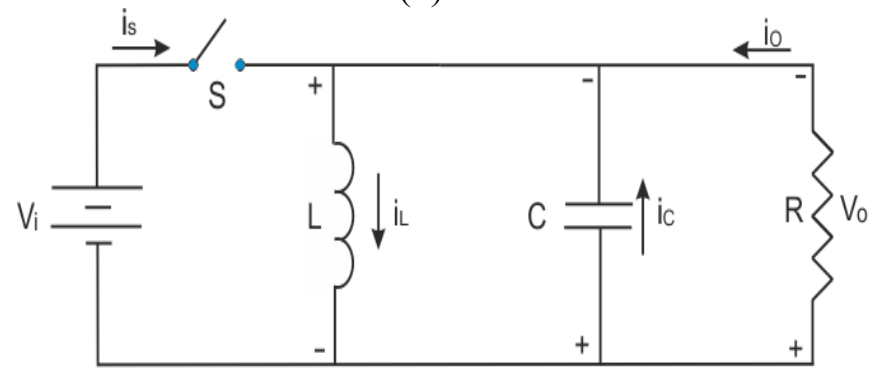

(c)

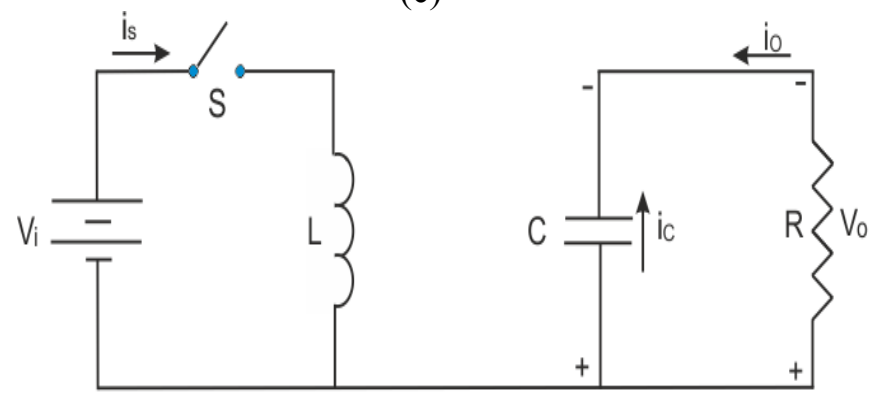

(d)

Fig. 1. (a) Buck-Boost DC-DC converter circuit diagram.

(b) On state equivalent circuit. (c) Offc state equivalent circuit. (d) Offnc state equivalent circuit.

\subsection{Switched space-state model}

Each state of the converter has a defined dynamics which can be described by means of a linear timeinvariant model (LTI) in state-space variables. In matrix notation:

$$
\begin{aligned}
& \dot{x}(t)=\mathbf{A} x(t)+\mathbf{B} u(t) \\
& y(t)=\mathbf{C} x(t)
\end{aligned}
$$

In equation (1), $x(t)$ is the vector of state-space variables, $u(t)$ the vector of input variables, $y(t)$ the vector of output variables, $\dot{x}(t)=d x(t) / d t$, is the vector containing the derivatives of the state-space variables, and A, B, C, D are matrices whose elements contains the electrical parameters of the circuit. In our case, we are going to consider a choice of state space variables with a physical meaning, that is, the current in the inductor $i_{L}$ and the voltage in the capacitor $\mathrm{v}_{\mathrm{C}}$ :

$$
\left\lfloor\begin{array}{l}
x_{1} \\
x_{2}
\end{array}\right\rfloor=\left\lfloor\begin{array}{l}
i_{L} \\
v_{C}
\end{array}\right\rfloor
$$

The input variables are:

$$
\left\lfloor\begin{array}{l}
u_{1} \\
u_{2}
\end{array}\right\rfloor=\left\lfloor\begin{array}{l}
V_{i} \\
i_{o}
\end{array}\right\rfloor
$$

The system output variables $y(t)$ coincide, in this case, with the state variables.

Each state of the converter has a particular equivalent circuit with their associated differential equations. Therefore, exist three different sets of matrix A B, one for each of them. Equations (4), (5) and (6) show the A B matrices for the On state, the Offc state and the Offnc state respectively:

$$
\begin{gathered}
\left\{\begin{array}{l}
\frac{d i_{L}}{d t}=\frac{V_{i}}{L} \\
\frac{d v_{c}}{d t}=-\frac{i_{o}}{C}
\end{array}\right. \\
\Rightarrow \mathbf{A}_{\mathbf{1}}=\left[\begin{array}{ll}
0 & 0 \\
0 & 0
\end{array}\right] \quad \mathbf{B}_{\mathbf{1}}=\left[\begin{array}{ll}
\frac{1}{L} & 0 \\
0 & -\frac{1}{C}
\end{array}\right]
\end{gathered}
$$




$$
\begin{gathered}
\left\{\begin{array}{l}
\frac{d i_{L}}{d t}=-\frac{v_{c}}{L} \\
\frac{d v_{c}}{d t}=\frac{i_{L}}{C}-\frac{i_{o}}{C}
\end{array}\right. \\
\Rightarrow \mathbf{A}_{2}=\left[\begin{array}{cc}
0 & -\frac{1}{L} \\
\frac{1}{C} & 0
\end{array}\right] \quad \mathbf{B}_{2}=\left[\begin{array}{ll}
0 & 0 \\
0 & -\frac{1}{C}
\end{array}\right] \\
\Rightarrow \mathbf{A}_{1}=\left[\begin{array}{ll}
\frac{d i_{L}}{d t} & =0 \\
\frac{d v_{c}}{d t} & 0 \\
0 & 0
\end{array}\right] \quad \mathbf{B}_{2}=\frac{i_{o}}{C} \\
\left.0 \begin{array}{ll}
0 & 0 \\
0 & -\frac{1}{C}
\end{array}\right]
\end{gathered}
$$

\section{Converter hybrid model}

The main characteristics of the Statecharts and their application to the hybrid model of the buck-boost DC-DC converter are presented in this section.

\subsection{Statechart characteristics}

Statecharts were introduced by D. Harel [13] and are a generalization of the event-driven finite state machine. A Statechart is made up of graphic elements (charts, states, transitions and unions) and text elements (data and events).

\subsection{Statechart components}

States: The Chart contains the states of the reactive subsystem, each one with its name and representing the operating modes of said subsystem in view of events. In the case of the Buck-Boost DC-DC converter, the previously analyzed possible states are: On, Offc and Offnc. The Offc and Offnc states operate in exclusive OR mode, so they can be part of a superstate or Off parent state. When the activity is passed to the parent state, the system will work in one of the child states, not both.

Data: The Statechart has different types of data associated with it: input and output to Simulink, local, constant, temporal and those that allow the use of the MATLAB workspace.

To interact with the Simulink model, the following data were considered:
-Simulink input data: ton: Boolean variable that represents the switch control signal; iL: variable that represents the current in the inductor.

-Output data to Simulink: swa, swb: integer selection variables of the continuous subsystem matrices; is: Input current.

Actions: The Stateflow syntax allows specifying (after the state name, in text form) the actions to be executed when entering (enter :), during (during :), when exiting the active state (exit :), or when occur an event. Other actions are the change of the value of outputs to Simulink such as swa and swb.

Transitions: In the Chart, the states are united by transitions. A transition is a graphical object that describes the circumstances (events and conditions) that cause the system to move from one state to another. Each transition is represented graphically with an arrow and can have a text, with three optional fields: event, condition and action. Between exclusive OR states there must always be a special transition called the default transition that specifies which of the states will be the active one at different hierarchical levels.

In our case, the transitions between the On and Off states are triggered when the Boolean condition expressed in braces is verified as true. For the conditional relation, the input data from Simulink ton was taken. When the simulation starts, the system goes to the On state by default, then if ton takes the value 0 , it goes to the Off state. From any of the substates of the superstate Off, it will go to the On state when the condition ton equal to 1 is satisfied.

When the Off state is active, the default transition passes the activity to the Offc state. Offnc will be active only if the conditional relationship determined by the value of the current in the inductor $\mathrm{i}_{\mathrm{L}}$, is less than or equal to zero.

Table 1 shows the link between transitions and states, where the chart is represented by the character '/', and each level in the hierarchy of states is separated by the character '. '.

\begin{tabular}{|c|c|c|}
\hline Transition & Original state & Destination state \\
\hline$[$ ton $==0]$ & $/$ On & /Off.Offc \\
\hline$[$ ton $==1]$ & $/$ Off & /On \\
\hline$\left[i_{L}<=0\right]$ & /Off.Offc & /Off.Offnc \\
\hline
\end{tabular}

Table 1. Links between transitions and states.

\subsection{Statechart triggering}

Simulink can "wake up" the Chart to an inherited or specified frequency, using a signal as a trigger or using a Statechart to drive the activity of another Statechart. If the transition does not carry any text, 
then it will trigger automatically, as long as any event occurs in the system. In the default mode of Inherited update of the Statechart, it is the Simulink inputs that determine when the chart will trigger during the Simulation. If input events are defined, the Stateflow block will be explicitly triggered by that signal on the chosen edge. In the model presented, the default mode was used, so the chart block is triggered in each simulation step (discrete or continuous) of the Simulink model.

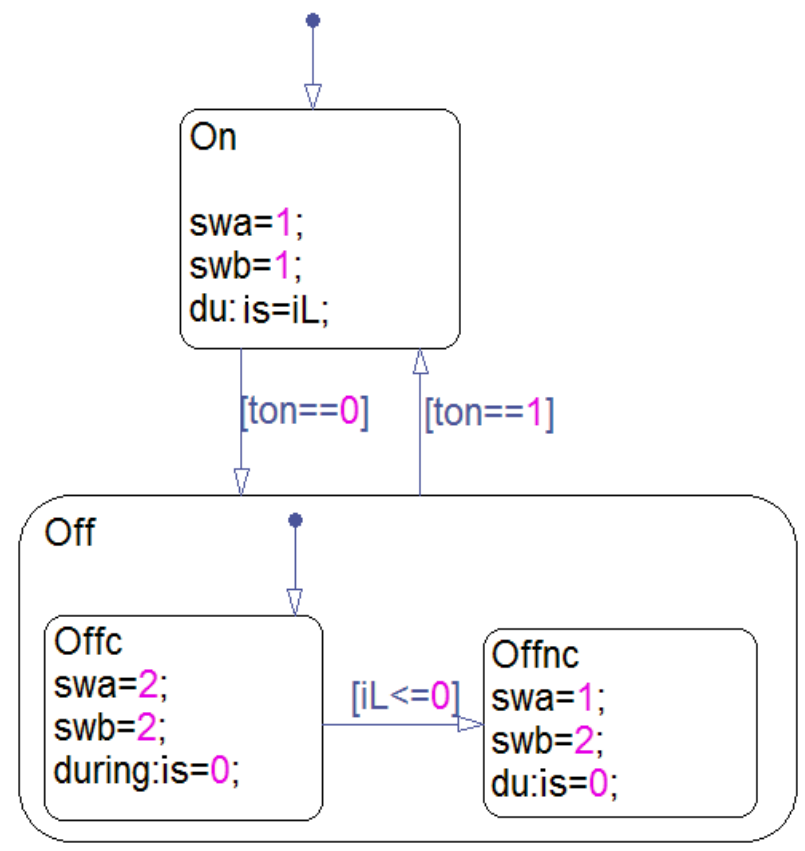

Fig. 2. Statechart of the buck-boost DC-DC converter.

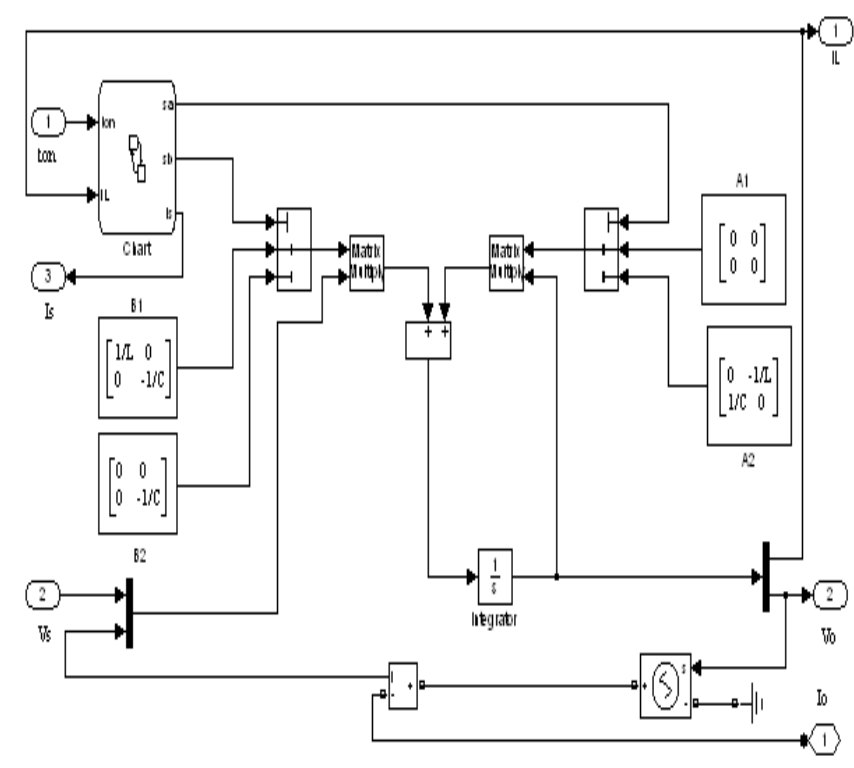

Fig. 3. Internal detail of the Simulink building-block for buck-boost DC-DC converter simulation.
The Statechart of the Buck-Boost DC-DC converter is shown in figure 2. The continuous part of the Hybrid System is implemented with the help of controlled switches, which select the matrices A B that describe the dynamics of each state. To make the converter model more versatile, it was configured as a building-block compatible with the SimPowerSystems library of the Simulink package. For this reason, a $V_{0}$ output terminal was is implemented, so that an electrical circuit element (load resistor) can be connected to it. The buildingblock internals can be appreciated in figure 3 .

\section{Simulation results}

The validation of the converter model and an application example of the developed buildingblock are presented in this section.

\subsection{Building-block validation in open loop operation (constant duty cycle).}

The buck-boost DC-DC converter can operate in continuous conduction mode (CCM) or discontinuous conduction mode (DCM). In CCM the converter has only two states: On and Offc. In DCM, it has an additional sate, the Offnc. If the switching frequency $f_{S}$ and the value of $L$ are kept constant, the load resistance $\mathrm{R}$ is the parameter who determines the conduction mode in which the converter is operating. There is a critical load resistance value $\left(\mathrm{R}_{\text {crit }}\right)$ that determines the boundary between both modes of operation and can be determined by equation (7) [14].

$$
R_{c r i t}=\frac{2 f_{s} L}{(1-D)^{2}}
$$

In (7), $\mathrm{D}=\mathrm{t}_{\mathrm{on}} / \mathrm{T}$ is the switch duty cycle, with $\mathrm{T}=1 / \mathrm{f}_{\mathrm{S}}$ being the switching period. The mean value of the steady-state output voltage $\mathrm{V}_{\mathrm{O}}$ of an ideal (without any power losses) buck-boost DC-DC converter can be computed using equations, obtained applying basic power conversion principles, that can be found in classical books as [14]. The equation (8) is valid for operation in CCM and equation (9) is valid for operation in DCM:

$$
\begin{aligned}
& C C M: R<R_{\text {crit }} \Rightarrow V_{O}=V_{S} \frac{D}{1-D} \\
& D C M: R>R_{\text {crit }} \Rightarrow V_{O}=V_{S} D \sqrt{\frac{R}{2 f_{s} L}}
\end{aligned}
$$


The presented hybrid model does not include parasitic elements (although they can be included easily, modifying the matrices of each state appropriately) and was obtained considering and ideal switch and diode. Therefore, it is expected that the simulation results will give output voltage values consistent with analytical calculations performed using (8) or (9), as appropriate for each conduction mode. Simulations of a converter operating in open loop (constant D) were carried out in the MATLAB ${ }^{R} /$ Simulink environment using the block proposed as an additional element of the SimPowerSystems library. The values of the converter parameters used in the calculations are given in Table 2.

\begin{tabular}{|c|c|}
\hline Parameter & Numerical value \\
\hline$C$ & $2 \mu \mathrm{F}$ \\
\hline$L$ & $0.25 \mathrm{mH}$ \\
\hline$f_{s}$ & $100 \mathrm{KHz}$ \\
\hline$V_{s}$ & $255 \mathrm{~V}$ \\
\hline
\end{tabular}

Table 2. Numerical values of the buck-boost DC-DC converter parameters used in the calculations and simulations.

\begin{tabular}{|c|c|c|c|}
\hline \multirow{2}{*}{$\mathrm{D}$} & \multicolumn{3}{|c|}{ Vo [V] steady-state } \\
\cline { 2 - 4 } & $\begin{array}{c}\text { Calculated } \\
\text { using (8) }\end{array}$ & $\begin{array}{c}\text { Simulation } \\
\text { result }\end{array}$ & $\begin{array}{c}\text { Error } \\
{[\%]}\end{array}$ \\
\hline 0.125 & 36.42 & 36.41 & -0.03 \\
\hline 0.250 & 85.00 & 84.79 & -0.25 \\
\hline 0.500 & 255.00 & 254.45 & -0.21 \\
\hline 0.750 & 765.00 & 764.29 & -0.10 \\
\hline 0.875 & 1785.00 & 1784.80 & $-0,01$ \\
\hline
\end{tabular}

Table 3. Comparison between the converter output voltages obtained by calculations and by simulation (in steady-state and CCM).

\begin{tabular}{|c|c|c|c|}
\hline \multirow{2}{*}{$\mathrm{D}$} & \multicolumn{3}{|c|}{ Vo [V] steady-state } \\
\cline { 2 - 4 } & $\begin{array}{c}\text { Calculated } \\
\text { using (9) }\end{array}$ & $\begin{array}{c}\text { Simulation } \\
\text { result }\end{array}$ & $\begin{array}{c}\text { Error } \\
{[\%]}\end{array}$ \\
\hline 0.125 & 266.68 & 267.30 & +0.23 \\
\hline 0.250 & 533.37 & 533.07 & -0.05 \\
\hline 0.500 & 1066.74 & 1064.30 & $-0,23$ \\
\hline 0.750 & 1600.11 & 1596.10 & -0.25 \\
\hline 0.875 & 1866.79 & 1865.30 & -0.08 \\
\hline
\end{tabular}

Table 4. Comparison between the converter output voltages obtained by calculations and by simulation (in steady-state and DCM).

In the first instance, the converter was simulated operating in continuous conduction mode (CCM) and then the value of the load resistance was altered so that the converter starts operating in discontinuous conduction mode (DCM). Table 3 shows the calculated steady-state values of $\mathrm{V}_{\mathrm{o}}$ using equation (8) and the ones obtained by simulation for different $\mathrm{D}$ values, using an $\mathrm{R}=50 \Omega$, which guarantees operation in CCM over the entire range of $\mathrm{D}$ considered. On the other hand, Table 4 shows the calculated steady-state values of $\mathrm{V}_{\mathrm{o}}$ using equation (9) and the ones obtained by simulation, for different values of $\mathrm{D}$, using an $\mathrm{R}=3500 \Omega$, which guarantees operation in DCM over the entire range of $\mathrm{D}$ considered. It can be seen that, for both operating modes (CCM and DCM), the steady-state values of $\mathrm{V}_{\mathrm{o}}$ obtained by simulation were very close (the worst case error is $0.25 \%$ ) to those obtained by theoretical analytical calculations.

\subsection{Application example in closed loop operation.}

The proposed building block can be considered as a simulation tool for buck-boost DC-DC converters in any application that does not require a detailed examination of the waveforms during the switch and diode commutation process.

As a typical example, a buck-boost DC-DC converter with PWM control has been selected, taken from the classical technical literature [15]. The converter has an internal loop to control the average input current $\mathrm{I}_{\mathrm{S}}$, the reference of which is provided by an external loop that regulates the output voltage $\mathrm{V}_{\mathrm{o}}$. In figure 4 only the current control loop is shown.

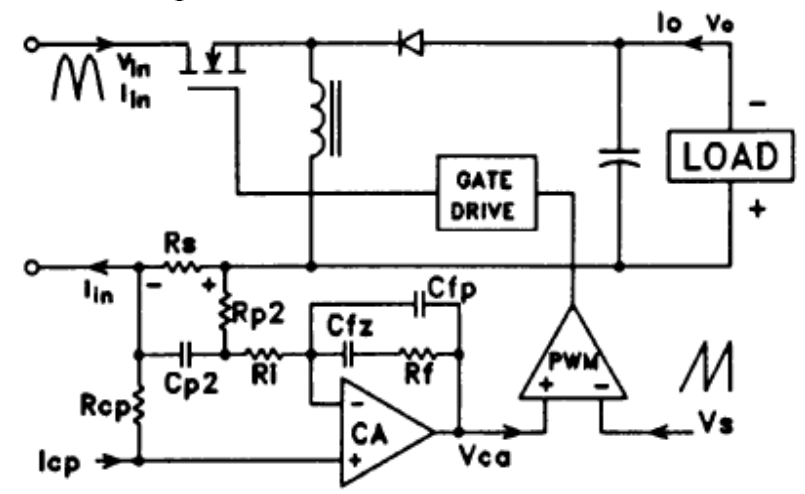

Fig. 4. Circuit schematic of a DC-DC buck-boost converter with average input current control loop. Taken from Ref [15].

With the exception of the input voltage, the values of the converter parameters used in the closed loop simulations are the same as those used in the openloop model validation. An input current sensing resistor is included, with a value $\mathrm{R}_{S}=0.025 \Omega$. The PWM modulator was implemented in a traditional way, comparing the controller output signal with a 
sawtooth signal of amplitude $\mathrm{V}_{\mathrm{SW}}=5 \mathrm{~V}$. The input current loop controller was modeled using the transfer function.

$$
G_{C i}(s)=K_{C i} \frac{\left(1+s / s_{z}\right)}{s\left(1+s / s_{p 1}\right)\left(1+s / s_{p 2}\right)}
$$

In $\quad(10) \quad \mathrm{K}_{\mathrm{Ci}}=37.103, \mathrm{f}_{\mathrm{Z}}=8 \mathrm{KHz}, \mathrm{f}_{\mathrm{pl}}=18.7 \mathrm{KHz}$, $\mathrm{f}_{\mathrm{p} 2}=100 \mathrm{KHz}$ are the frequencies of the zeros and poles of the current controller. There are saturations limits at $0.5 \mathrm{~V}$ and $4.5 \mathrm{~V}$.

The PI controller, used for the regulation of the output voltage, was modeled by the following transfer function:

$$
G_{C v}(s)=K_{P}+K_{I} / s
$$

With: $K_{P}=1.10-3 ; K_{l}=12$. It has saturations limits at 0 and 1.

The Simulink diagram of the complete system used in the simulations is shown in figure 5. In this figure you can see the building-block containing the hybrid model of the DC-DC converter (developed in the previous sections) and, around it, the internal loop for the control of the average input current and the external loop (PI controller) for the regulation of the output voltage.

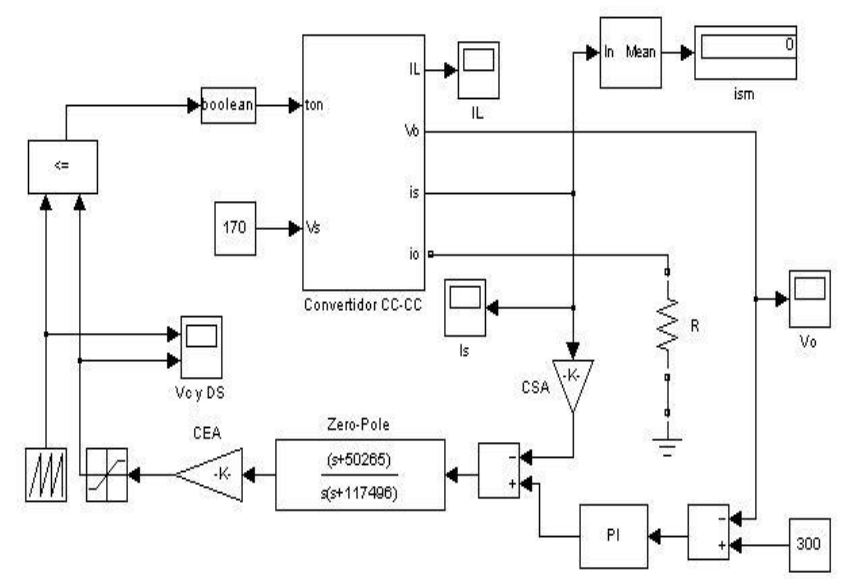

Fig. 5. Simulink block diagram of the DC-DC buck-boost converter model and the control loops.

A working condition defined by the following values was simulated: $\mathrm{V}_{\mathrm{S}}=170 \mathrm{~V} ; \mathrm{V}_{\mathrm{o}}=300 \mathrm{~V}$; $\mathrm{P}_{\mathrm{o}}=1 \mathrm{KW}(\mathrm{R}=90 \Omega) ; \mathrm{Cp}_{2}=0\left(\mathrm{Sp}_{2}=\infty\right)$. Figure 6 shows the great similarity between the waveforms obtained by simulation and the real ones, which were extracted from [15]. Figure 6 (a) and (b) show the controller output voltage, real and simulated respectively. In figure 6 (c) and (d) the input current of the converter, real and simulated, is presented. The mean value of the input current was determined, and the value obtained, $\mathrm{I}_{\mathrm{S}}=5.884 \mathrm{~A}$, was equal to the given in the aforementioned reference.

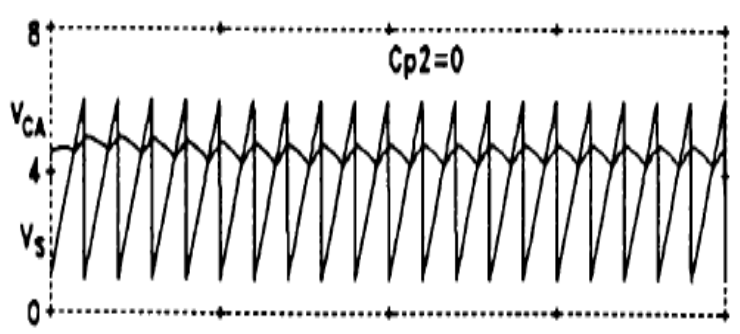

(a)

Diente de Sierra / Tensión de Salida del Compensador

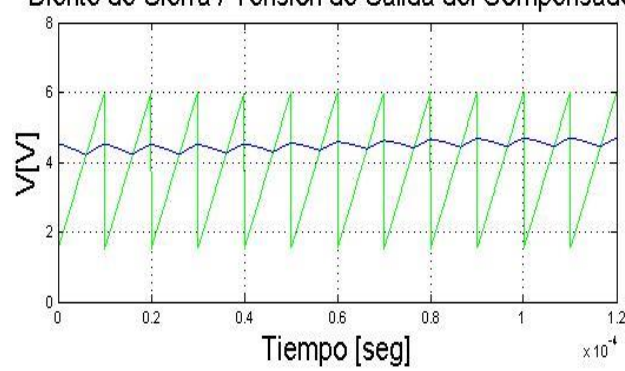

(b)

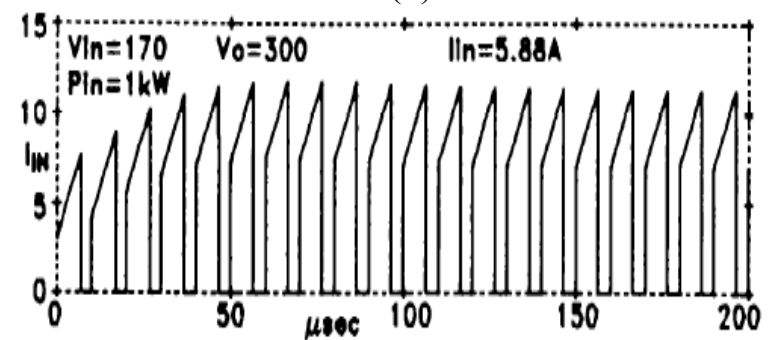

(c)

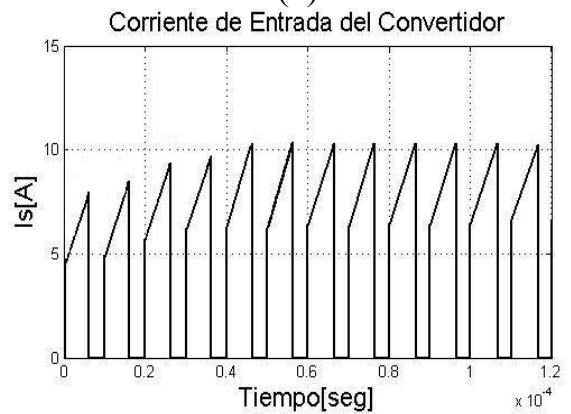

(d)

Fig. 6. Closed loop operation of the buck-boost DC-DC converter. Controller output voltage: (a) actual, (b) simulation result. Converter input current: (c) actual, (d) simulation result.

\section{Conclusions}

This article presented an implementation of the hybrid model for the Buck-Boost DC-DC converter, using the Statecharts technique, written in Stateflow language. The model was implemented as a building block and has been designed to facilitate its integration into existing Simulink models of more 
complex systems that incorporate elements of the SimPowerSystems library.

The building block performance was validated in open loop operation by comparing simulation results with analytical calculations in both conduction modes (CCM and DCM). In addition, an example of application of the proposed block was shown, consisting in the simulation of a converter with average input current control (for PFC applications). In both cases, a correct performance with a minimal discrepancy was corroborated.

The developed building block has the following salient characteristics:

- Correctly represents the operation of the converter throughout the entire operating range and in both operation modes (CCM and DCM).

- It can be simulated using fixed step numerical integration routines, with simulation step not as reduced as required by other models, since details of the switch commutation process are not represented.

- The convergence problems of the numerical integration routines are minimized.

- When the block is included in a more complex model that have other subsystems with time constants slower than that of the converter itself, a significant temporal evolution of the slower subsystem can be observed using a reasonable computation time.

- It allows to carry-out, in an agile and fast way, repeated simulations in stability studies of closed loop systems.

- Additional elements (in order to represent converter power losses and other parasitic elements) can easily be included in the proposed model modifying adequately the matrices $\mathrm{A}$ and $\mathrm{B}$ of the sates On, Offc and Offnc.

\section{Acknowledgment}

The authors express their gratitude to the Argentine institutions UNLP, CONICET and ANPCyT, since without their support this work would not have been possible.

\section{References:}

[1] Cadence Design Systems, Inc., San José, California, USA, 2021. http://www.pspice.com.

[2] Powersim Inc. 2275 Research Blvd, Suite 500 Rockville, MD 20850, U.S.A., 2021. https://www.powersimtech.com.

[3] G. Herbst, "A Building-Block Approach to State-Space Modeling of DC-DC Converter Systems", J, vol. 2, no. 3, pp. 247-267, Jul. 2019.

[4] Yu-Jun Mao, Chi-Seng Lam, Sai-Weng Sin, Man-Chung Wong, Rui Paulo Martins,
"Review and Selection Strategy for HighAccuracy Modeling of PWM Converters in DCM", Journal of Electrical and Computer Engineering, vol. 2018, 16 pages, 2018.

[5] O. A. Beg, H. Abbas, T. T. Johnson and A. Davoudi, "Model Validation of PWM DC-DC Converters," in IEEE Transactions on Industrial Electronics, vol. 64, no. 9, pp. 7049-7059, Sept. 2017, doi: 10.1109/TIE.2017.2688961.

[6] J. A. Flórez-Vargas y R. Alzate Castaño, «Power converter circuits: A hybrid dynamical case», DYNA, vol. 84, no. 200, pp. 143-150, ene. 2017.

[7] Z. Karami, Q. Shafiee, S. Sahoo, M. Yaribeygi, H. Bevrani and T. Dragicevic, "Hybrid Model Predictive Control of DC-DC Boost Converters With Constant Power Load," in IEEE Trans. on Energy Conversion, vol. 36, no. 2, pp. 1347-1356, June 2021.

[8] Hejri, M., "Global Hybrid Modeling and Control of a DC-DC Buck-Boost Converter via Mixed Logical Dynamical Systems". NASHRIYYAH -I MUHANDISI -I BARQ VA MUHANDISI -I KAMPYUTAR -I IRAN, AMUHANDISI -I BARQ, 17(1 ), pp.1-12. 2019.

[9] Wang, S., Wu, X., Yang, Y., Zhu, C., Wu, Z., \& Xia, C. "Hybrid modeling and control of ICPT system with synchronous three-phase triple-parallel Buck converter". Wireless Power Transfer, 7(1), 10-18. (2020)

[10] Liangyu Huang, Yimin Lu, "Hybrid Modeling Method and Bifurcation Characteristics Analysis for Buck Converter with Constant Power Load", Hindawi Mathematical Problems in Engineering, vol. 2019, 10 pages.

[11] C. Albea, G. Garcia and L. Zaccarian, "Hybrid dynamic modeling and control of switched affine systems: Application to DC-DC converters", 2015 54th IEEE Conference on Decision and Control (CDC), 2015, pp. 22642269.

[12] MATLAB Stateflow User's Guide, Mathworks Inc., MA, USA, 2015.

[13] Harel, D., Statecharts: A Visual Formalism for Complex Systems, Science of Computer Programming, Volume 8, Issue 3, 1987, Pages 231-274.

[14] Erickson, R., D. Maksimovic, Fundamentals of Power Electronics, 3rd Edition, Spinger, Switzerland (2020).

[15] Dixon, L., "Average Current Mode Control of Switching Power Supplies," Unitrode Power Supply Design Seminar SEM700, pp. 3-356-3369 (1990).

Creative Commons Attribution License 4.0 (Attribution 4.0 International, CC BY 4.0)

This article is published under the terms of the Creative Commons Attribution License 4.0

https://creativecommons.org/licenses/by/4.0/deed.en_US 